\title{
A Dual Cylindrical Tunable Laser based on MEMS
}

\author{
Ahmed Fawzy \\ Department of Electrical Engineering \\ Minia University \\ Nanotechnology central lab \\ Electronic Research Institute \\ Cairo, Egypt
}

\author{
Osama M. EL-Ghandour \\ Department of Elect., Commu., and \\ Comp. Engineering \\ Helwan University \\ Cairo, \\ Egypt
}

\author{
Hesham F.A. Hamed \\ Department of Electrical \\ Engineering \\ Minia University \\ Minia, \\ Egypt
}

\begin{abstract}
Free space optics is considered the topic of the day and have a large variety of applications which free space separates source from destination such as External cavity tunable laser (ECTL). In ECTL, laser source emits Gaussian beam that propagates in plane with substrate until reach external reflector. The efficiency of these applications depends on the amount of light that coupled back to the laser, called coupling efficiency. Increasing coupling efficiency depends on using assembled lens's or any optical part in the path between laser front facet and external reflector, which result in increasing the cost and integration effort. We innovate here anew configuration of external cavity tunable laser based on cylindrical (curved) Mirrors. The usage of cylindrical mirror affects on the amount of light that coupled back to laser and that decreases the alignment requirement in the laser assembly as compared to another configurations based on flat mirror. The fabrication of cylindrical mirror is simple with respect to spherical mirror so it can be used in batch fabrication. Tuning achieved by using micro electro mechanical system MEMS technology. The system consists of a laser cavity and a two filter cavities for wavelength selection. The formation of cylindrical microstructures were made into the substrate volume. So we report also the micromachining method that used for fabricating the cylindrical mirror. Anisotropic etching and the deep reactive ion etching (DRIE) are especially useful for the batch fabrication of large optical mechanical devices. The characteristics of the laser's spectral response versus laser facet reflectance variations are described via simulations. The diffraction of light in ECTL formed by the laser front facet and the external reflector are taken into account. Here we report all things about the model including the fabrication steps and simulation analysis.
\end{abstract}

Keywords-Dual ECT; wavelength tuning; MEMS; DRIE

\section{INTRODUCTION}

Optical communications are the most important branch in communication engineering field as it introduces high speed communication, high bandwidth and low interference. Tunable laser will be discussed as one of the most important topic in optical communication[1-7]. We report here ECTL as a main stream technology in a tunable laser due to its advantages of simple configuration, small device dimensions, high tuning speed, wide tuning range, spectrum purity and high power. There are types of external cavities introduces wide tuning range but suffers from large device dimensions such as Littrow and Littman configurations which the tuning is achieved by rotating and translating an external grating simultaneously[89]. The rapid progress of the MEMS technology allows for miniaturization of a bulky tunable laser, improvement of the tuning speed and the mechanical reliability at lower fabrication cost. Designs based on the simple ECTL with a movable external mirror suffer from the tradeoff between tuning range and side mode suppression SMS [2,3,11-13]. So we present a model that compensates between the tuning range and SMS taking into account the diffraction effects for the light between laser facet and external mirror, and tuning range calculations. The paper is organized as follows: in section II materials and fabrication methods is explained in detail. In section III, theoretical analysis is explained and show the effective reflectivity concept and how it is affected by curved mirror. In Section IV, the results which shows the difference between single ECTL and dual ECTL the wavelength shift in the optical output of the laser is calculated based on that calculation.

\section{MATERIALS AND FABRICATION METHODS}

ECTL uses Fabry perot (FP) filters which exists between laser facet and external reflector. The tunability achieved by using FP filter, which comprises of two reflectors one fixed and another movable separated by free space or air gap. The external reflectors (mirrors) made from crystalline silicon(CSI) for many reasons [14], First unique material properties of crystalline silicon in combination with wet anisotropic etching or advanced DRIE permit for the fabrication of improved optical components with mechanical parts. Second micromechanics needed some features in materials to provide its operation such as flat alignment, exact surface orientation and low oxygen content and low crystal defect density all of this exists in C-SI as well as unusual crystal cuts such as [110]silicon, So we consider silicon material the most important one in MEMS systems that used in optics and Commercially C-SI uses as the standard substrate for microelectronics.

C-SI has also mechanical properties useful for all optical application that depend on moving parts. Stress-strain behavior of C-SI is unique which the absence of plasticity for temperature under $700{ }^{\circ} \mathrm{C}$ help in stable operation of mechanical component. Young's modulus E of C-SI affected extremely with the crystal orientation .

There are many micromachining techniques used in MEMS[1, 14-15]. we report here the most important of them. Surface Micromachining (SM) which used in mirrors fabrication. we can consider SM as a direct extension of semiconductor manufacturing technology. SM can manufacture devices in order of 50 - 100 micrometer. The key in this technique is the use of sacrificial layer which this layer are usually SIO2. This technology is based on depositing and 
etching structure and sacrificial films. After deposition of thin film, sacrificial layer is etched away, leaving a completely assembled microstructure as shown in fig (1). By using of this technology free space components can be realized. The important applications of this technology are optical crossconnect switches [16-19] and micro-optical free-space benches. The main advantage for this technique that the components after fabrication become self-assembled. The functional layer in SM almost made from poly SI and this consider disadvantage in mirrors. Also maximum possible thickness of the microstructure is limited to that of the deposited film. The main problem in this technique is how the mirror lifted off from substrate to the desired position. Usually we use microprobes to do this action and due to this action the process needed more time and money.
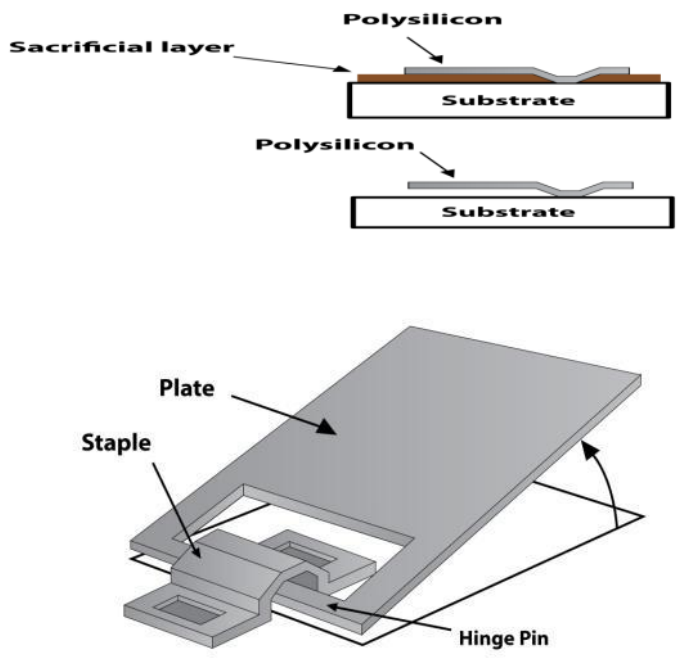

Fig. 1. General schematic diagram for the fabrication steps of micromirror by using SM

Second techniques Silicon-on-insulator (SOI) wafers consists of $\mathrm{SiO} 2$ sandwiched between two C-SI wafers. The sacrificial layer here is also $\mathrm{SiO} 2$ layer. Removing of sacrificial layers allows for mechanical movement of structures. Here the complete configuration can putted on chip that includes mirrors, actuator and fiber alignment structure as well as laser source assembled on chip and the fabrication of all components can made in a single DRIE etch process[20].

We introduce here a new monolithically dual silicon ECTL configuration based on SOI and DRIE with a standard FP semiconductor laser coupled with two ends with highly reflective movable curved mirror in one end and another end with fixed curved mirror as shown in fig (2)[15, 21-22]. The length of the gap between the laser and the movable mirror is varied by means of a actuator fabricated also on the same chip. The output of the laser is collected after transparent mirror. However spherical mirrors can be used, This paper concerned with curved mirrors only. This is because steps of fabrication of spherical mirrors cannot be used in batch fabrication. Also spherical mirror fabrication causes the wafer to be fragile due to long etching time. So our geometry depends on simplicity in fabrication as well as miniaturization [20].

Transparent Mirror

Reflective Movable Mirror

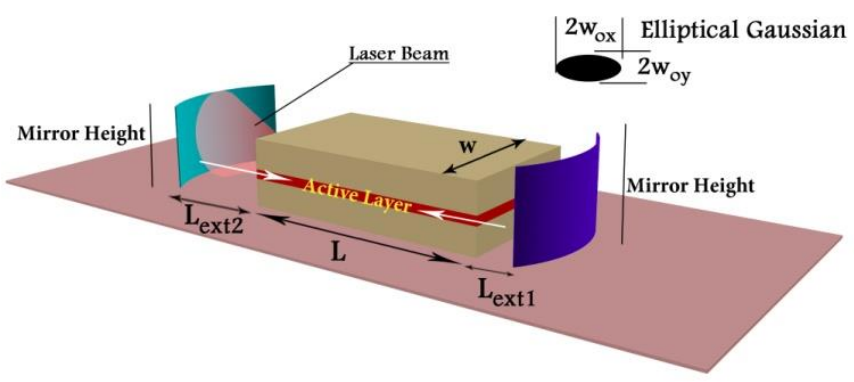

Dual External Cavity Semiconductor Laser

Fig. 2. General schematic diagram for the Dual ECTL source with external curved mirror

\section{THEORITICAL ANALYSIS}

The most important parameters in the ECTL designs are the effective reflectivity of the external cavities $r_{\text {eff }}$ and the optical cavity length $\mathrm{L}_{\mathrm{ext}}$. These parameters affects on the performance of FP filters which determined by free spectral range FSR, cavity finesse $\mathrm{F}$ and cavity filter bandwidth $\mathrm{BW}$. FSR can be defined simply as the spacing between adjacent maxima of a transmitted wavelength. FSR $=\lambda^{2} / 2 \mathrm{~L}_{\text {ext }}$ where $\lambda$ is operating wavelength. From this relation we can show that a shorter cavity provides wider FSR. F is determined also by the following relation $\mathrm{F}=\pi \sqrt{\mathrm{r}_{\text {eff }}} / 1-\mathrm{r}_{\text {eff }}$ that shows $\mathrm{F}$ depend only on $r_{\text {eff }}$. BW of the filter defined as the sharpness of each transmission peak $\mathrm{BW}=\mathrm{FSR} / \mathrm{F}$.

Now we will focus on another parameter that called mirror loss term $\alpha_{M}$ that depend also on $r_{\text {eff }}$ and $L_{\text {ext }}$.For FP laser $\alpha_{\mathrm{M}}$ can expressed as follow:

$$
\alpha_{\mathrm{M}}=-(1 / 2 \mathrm{~L}) \ln \left(\left|\mathrm{r}_{1}\right|\left|\mathrm{r}_{2}\right|\right)
$$

where $L$ is the length of the semiconductor laser and $r_{1}, r_{2}$ are the amplitude reflectances of the two facets of the laser. For external cavity, the effective reflectance is used to describe the effect of the external reflector [2, 11-12].

$$
r_{\text {eff }}(v)=r_{2}+\left(1-\left|r_{2}\right|^{2}\right) r_{\text {ext }} \exp \left(-j 2 \pi v \tau_{\text {ext }}\right)
$$

where $\tau_{\text {ext }}$ refers to the round trip delay through the external cavity of length (Lext), $v$ is the frequency and the $r_{\text {ext }}$ is the reflection of external reflector. Here we neglected multiple reflections in the external cavity for simplicity. The introduction of the external reflector can be thought to change the laser mirror reflectance $r$ either $r_{1}$ or $r_{2}$ by an amount that is proportional to the ratio of light coupling back to the laser to the light going out. Both the amplitude and the phase of the reflected light is changed. This can be described by replacing $\begin{array}{llll}r_{1} & \text { and } & r_{2} \text { by } r_{\text {eff1 }} & \text { and }\end{array}$ 
respectively that is obtained via a coherent superposition of $r$ of laser and the external reflectance. In other words The effective reflectivity is the coherent sum of the internal laser facet reflectance and the external ECTL reflectance, $r_{\text {eff }}=r_{\text {int }}+$ $r_{\text {ext }}$. The interference between the internal and external reflections on the laser facets usually the important part of the wavelength dependency of the effective reflectivity, and therefore is the basic reason for the wavelength selectivity in many ECTL lasers. $\alpha_{M}$ now can expressed as follow:

$$
\alpha_{\mathrm{M}}=-(1 / 2 \mathrm{~L}) \ln \left(\left|\mathrm{r}_{\text {eff } 1}\right|\left|\mathrm{r}_{\text {eff2 }}\right|\right)
$$

The external reflectance can be calculated by using the overlap integral at the facet of the reflected from external reflector after round trip $\psi_{\text {ext }}$ and the field emitted from FP laser chip $\psi_{G}$ by the following relation:

$$
\eta=\iint_{-\infty}^{\infty} \psi_{G}(x, y) \psi_{\text {ext }}^{*}(x, y) d x d y / \iint_{-\infty}^{\infty}\left|\psi_{\text {ext }}(x, y)\right|^{2} d x d y
$$

In our study, the emitted field from FP laser was taken to be a normalized elliptic Gaussian field, with waist dimensions corresponding to the output beam of the solitary laser.

$$
E(x, y)=E_{m} e^{\frac{-x^{2}}{w_{0 x}^{2}}+\frac{-y^{2}}{w_{0 y}^{2}}}
$$

where $E_{m}$ is the field amplitude at the center of the beam, $w_{\text {ox }}$ is the spot size in the $x$ direction and $w_{\text {oy }}$ is the spot size in the y direction. The laser spot size is 3.5 micrometer in the horizontal direction and 1 micrometer in the vertical direction, in an elliptical shape, the laser wavelength is 1550 $\mathrm{nm}$. The external reflector is considered to be curved mirror as shown in fig (3). The curved mirror not only reflect the beam but also add a phase due to the curvature of a mirror. The reflection coefficient of a curved mirror $R_{c}$ can be expressed as:

$$
\mathrm{R}_{\mathrm{c}}=\mathrm{R}_{\mathrm{m}} \mathrm{e}^{-2 \mathrm{j} \mathrm{k}_{\mathrm{o}}\left(\mathrm{d}_{0}-\left(\mathrm{R}-\sqrt{\left(\mathrm{R}^{2}-\mathrm{x}^{2}\right)}\right)\right)}
$$

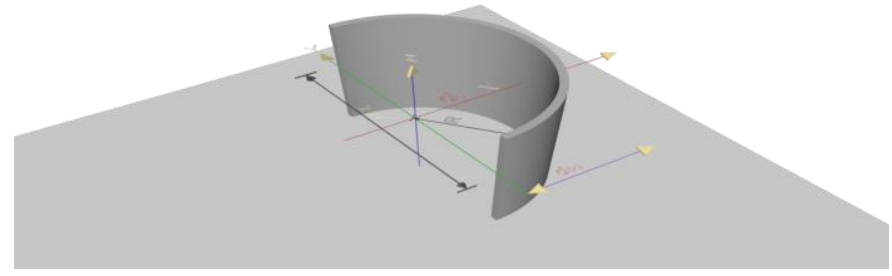

Fig. 3. 3D model of external curved mirror with radius of curvature $\mathrm{R}$ and mirror width $2 \mathrm{w}$

Thus for a mirror of reflection coefficient $R_{m}$, and a radius of curvature $R$ in the wafer plane where $d_{0}$ is the mirror sag at the plane of the wafer given by:

$$
\mathrm{d}_{0}=\mathrm{R}-\sqrt{\mathrm{R}^{2}-\mathrm{w}^{2}}
$$

with $2 \mathrm{w}$ the mirror width assumed to be $32 \mu \mathrm{m}$ in our calculations. By using the equations (2), (3), (4),(5) and(6) we can calculate the coupling power of external reflector when the distance between the laser and the micromirror surface was varied.
For the sake of comparison we use two kinds of mirror flat mirror and curved mirror as shown in fig(4).

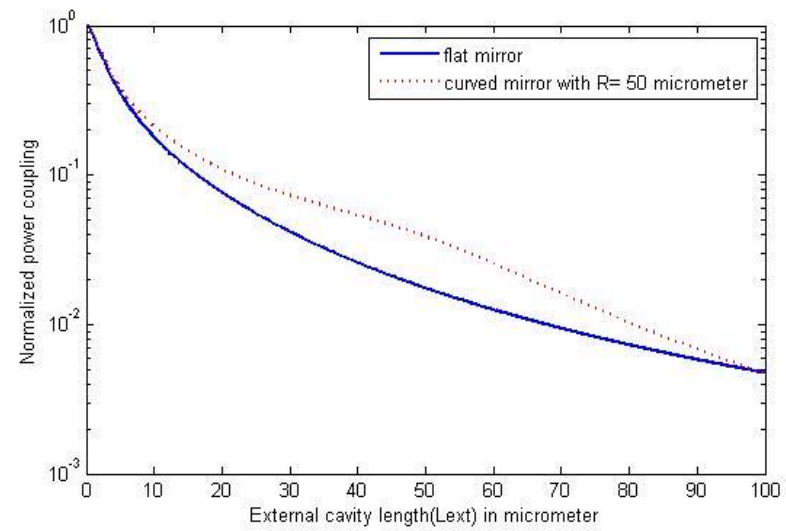

Fig. 4. Normalized power coupling efficiency of the curved micromirror with radius of curvature of 50 micrometer and flat micromirror versus External cavity tunable laser

In our model, a plane wave expansion method is used $[8,19]$. In this method the effect of transfer function of free space between laser facet and external reflector, called diffraction effect, is taken into account. As shown in figure (4), the curved mirror makes collimation for the light in one direction so the amount of light that coupled back has been increased with respect to the flat mirror. In other words, the amplitude and phase of effective reflectivity will affect on not only the power coupled to laser, but also the frequency shift. This is because the phase of round trip of laser cavity will be changed due to existence of external cavity. The change in the phase can be calculated from following equation:

$$
\Delta \emptyset_{\mathrm{l}}=2 \pi \tau_{\mathrm{l}}\left(\mathrm{U}-\mathrm{U}_{\mathrm{th}}\right)+\emptyset_{\mathrm{r}}-\alpha\left[\ln \left(\frac{1}{\left|\mathrm{r}_{\mathrm{eff} 1}\right|\left|\mathrm{r}_{\text {eff } 2}\right|}\right)-\ln \left(\frac{1}{\left|\mathrm{r}_{1}\right|\left|\mathrm{r}_{2}\right|}\right)\right]
$$

where $\tau_{1}$ is the round trip time delay of the optical beam inside the primary laser cavity, $\emptyset_{\mathrm{r}}$ represents the total phase of effective reflectivity due to two cavities, $U$ is the new oscillation frequency, $U_{\text {th }}$ is the oscillation frequency before using an external cavities and $\alpha$ is the linewidth enhancement factor in the semiconductor material. From this relation, we can find a new formula for the frequency shift of the laser emission that result due to using dual cavities as follows:

$$
\text { freq shift }=U-U_{\text {th }}=\left[\alpha\left[\ln \left(\frac{1}{\left|\mathrm{r}_{\text {eff } 1}\right|\left|\mathrm{r}_{\text {eff } 2}\right|}\right)-\ln \left(\frac{1}{\left|\mathrm{r}_{1}\right|\left|\mathrm{r}_{2}\right|}\right)\right]-\emptyset_{\mathrm{r}}\right] /
$$

This expression for the frequency shift is valid for both weak and strong feedback reflections. It can thus be used to study the effect of the diffraction on the frequency shift and hence on the resonance frequency of the laser. To calculate this frequency shift, it is required to evaluate both the amplitude and phase of the reflected beam after travelling in the external cavity. The phase difference and the balance between the amplitudes of the internal and external reflections play an important roles in many of the sensing application as shown in figure(5) [23-25]. 


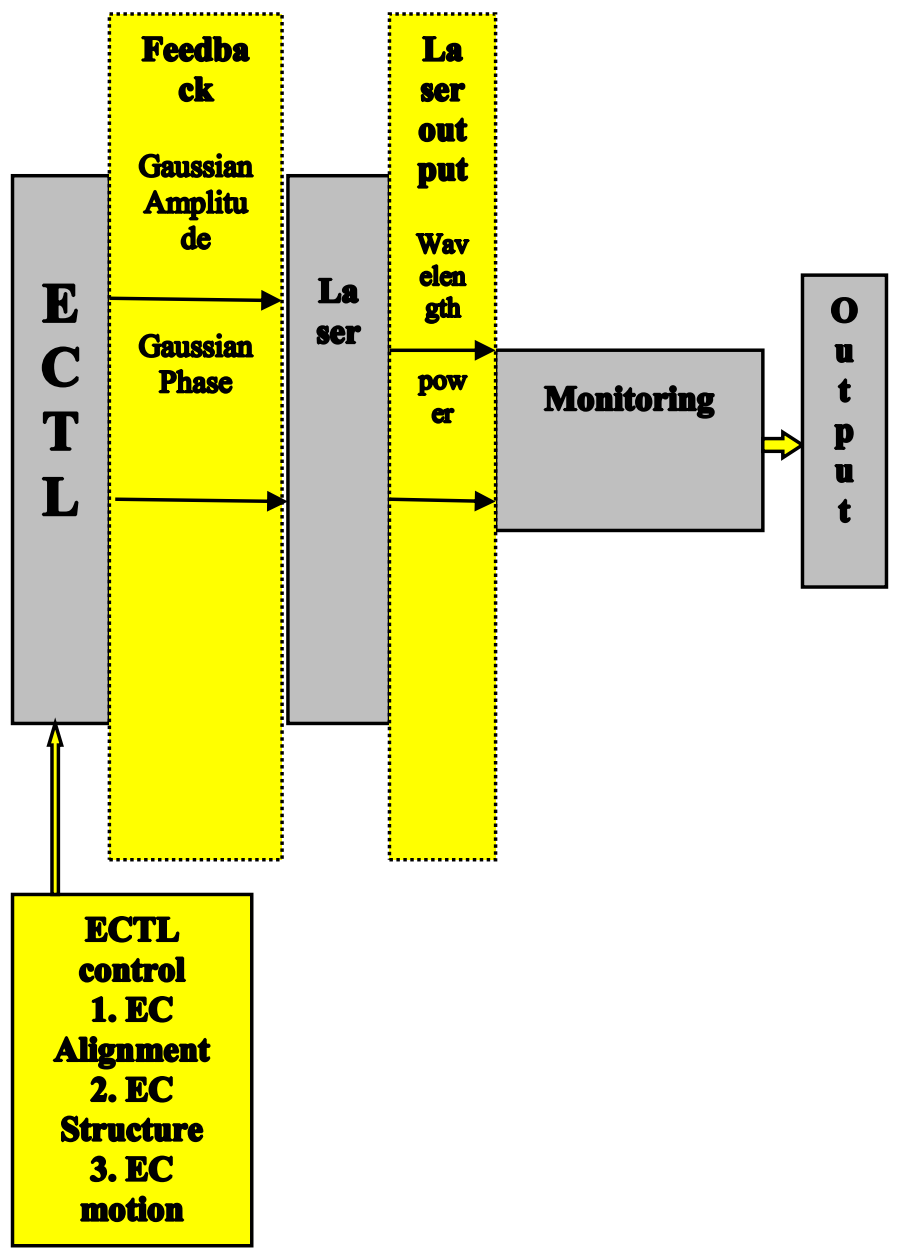

Fig. 5. General concept of sensing in ECTL

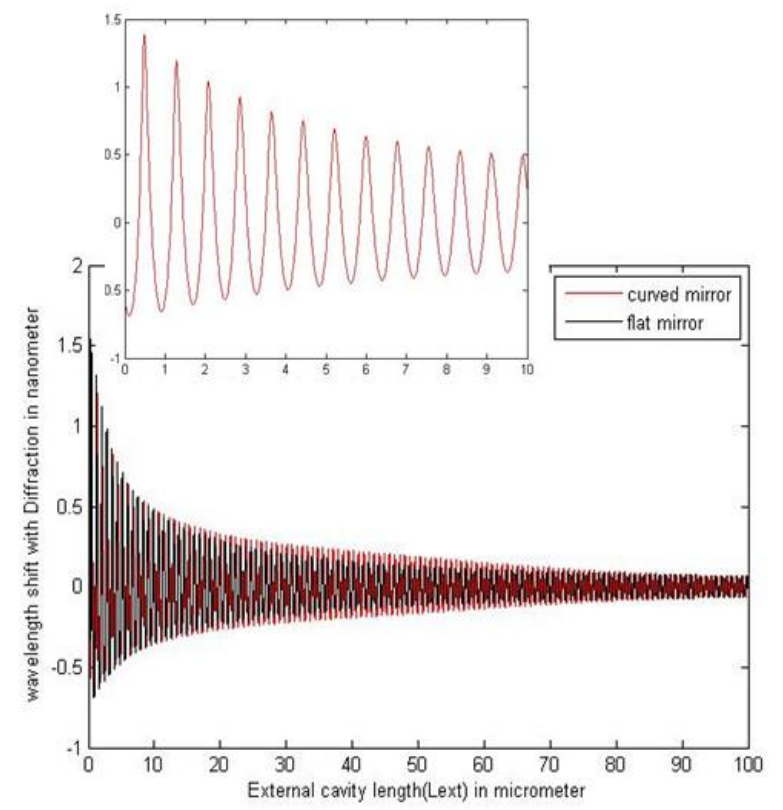

Fig. 6. Wavelength shift versus external cavity length of single ECTL which its external cavity is comprised by micromirror and the front facet of the semiconductor laser. When the micromirror flat (black line) and when the micromirror curved with radius of curvature 50 micrometer (red line)
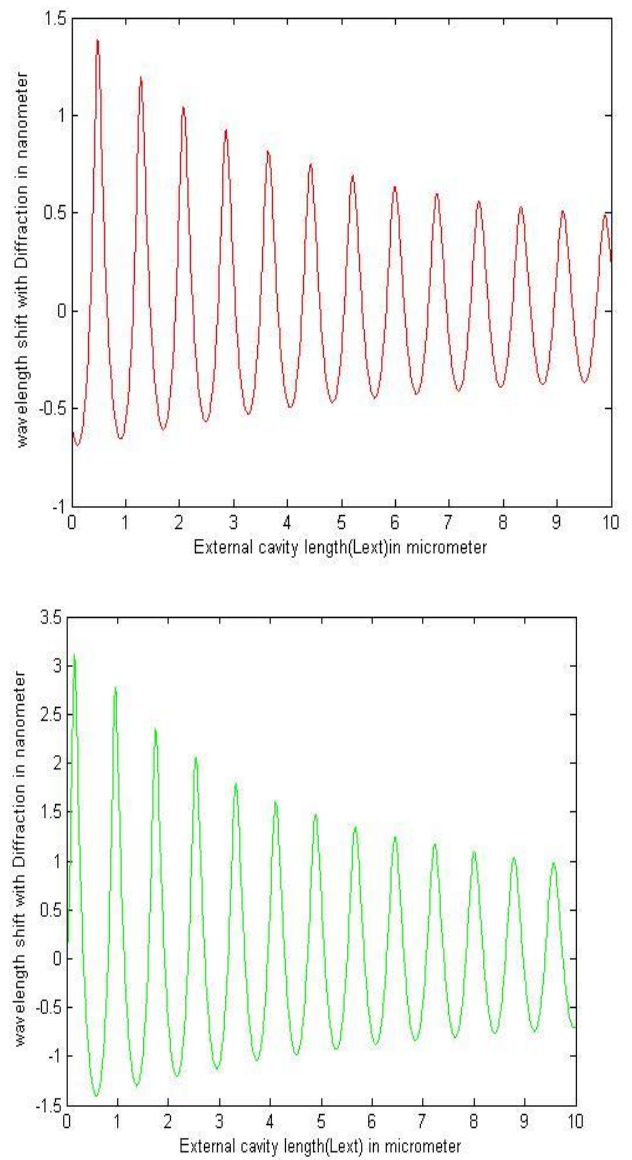

Fig. 7. Wavelength shift versus external cavity length is shown in (a) single ECTL formed by the external cavity comprised by curved micromirror and the front facet of the semiconductor (Red line) (b) Dual ECTL with two cavities formed by curved mirror in both sides. Radius of curvature of both cases $\mathrm{a}, \mathrm{b}=50$ micrometer and reflectivity of mirror $=.32$ (green line)

\section{RESULTS}

For a curved external mirror, the coupled power that come back to active cavity from external mirrors is affected by phase that is introduced by external mirror as shown in figure(6). For flat mirror there is no collimation as curved mirror; which makes collimation for light in one direction at the point of radius of curvature. The amount of power that coupled back from curved mirror is larger than the flat mirror. In a dual configuration, we assume only one round trip in the calculation after one round trip; the optical intensity may still be strong enough to perturb the operation of the laser. In figure 7 we see the difference in range of wavelength shift between single ECTL and dual ECTL. In case of a dual ECTL the range of wavelength shift is greater than the double of single ECTL.

\section{CONCLUSIONS}

In this paper, we propose a new simple unique model based on curved mirror. This model uses two cavities instead of single cavity. Our study reveals several important features that we utilized in the design of miniaturized MEMS tunable laser. These features include the importance of curved mirror which achieves efficient coupling of light than flat mirror. A micromachining method, that is used to fabricate curved 
mirror, is very simple comparable with spherical mirror so it can be used in batch fabrication. A new analytical approach is developed to determine the performance of dual ECTL taking into account diffraction effect. Analytical results show that dual ECTL can achieve larger tuning range than that of single cavity. All these factors make our model the best miniaturized tunable laser between all models.

\section{REFERENCE}

[1] O. Solgaard, A.A. Godil, R.T Howe, L.P. Lee, Y.-A. Peter and H. Zappe, "Optical MEMS: From Micromirrors to Complex Systems," Journal of Microelectromechanical Systems, vol.23, no.3, pp.517-538, June 2014

[2] A. Q. Liu," Photonic MEMS Devices Design, Fabrication and Control", CRC Press, 2009.

[3] A. Q. Liu, and X. M. Zhang, "A review of MEMS external-cavity tunable lasers", Journal of Micromechanics and Microengineering, Vol. 17, No. $1,2007$.

[4] O. Solgaard, "Photonic Microsystems: Micro and Nanotechnology Applied to Optical Devices and Systems",Springer New York, 2009.

[5] M. Ren, H. Cai, Y. D. Gu, P. Kropelnicki, A. B. Randles and A. Q. Liu, "A tunable laser based on nano-opto-mechanical system", 27th IEEE International Conference on Micro Electro Mechanical Systems (MEMS 2014), San Francisco.

[6] Komljenovic, T., Srinivasan, S., Davenport, M., Norberg, E., Fish, G., Bowers, J.E." Widely-tunable narrow-linewidth lasers with monolithically integrated external cavity", Conference on Lasers and Electro-Optics (CLEO), 2015.

[7] W. M. Zhu, W. Zhang, H. Cai, J. Tamil, B. Liu, T. Bourouina, and A. Q. Liu "A MEMS Digital Mirror for Tunable Laser Wavelength Selection", International Conference on Solid-State Sensors, Actuators and Microsystems, pp. 2206-2209, 2009.

[8] A. Q. Liu, X. M. Zhang, D. Y. Tang, and C. Lu, "Tunable laser using micro machined grating with continuous wavelength tuning", Applied Physics Letters, Vol. 85, No. 17, pp. 3684-3686, 2004.

[9] X. M. Zhang, A. Q. Liu, D. Y. Tang, and C. Lu, "Discrete wavelength tunable laser usingmicroelectromechanical systems technology", Appl. Phys. Lett., Vol. 84, pp 329, 2004.

[10] X. M. Zhang, A. Q. Liu, C. Lu and D. Y. Tang, "A real pivot structure for MEMS tunable lasers," IEEE Journal of Microelectromechanical Systems, Vol. 16, no. 2, pp. 269-278, 2007.

[11] A., Fawzy, S., El-sabban, , I., Ismail and D., Khalil," On the Modeling of an External Cavity Tunable Laser ECTL Source with Finite Mirror Dimensions", Progress in Electromagnetic Research Symposium Proceedings, Stockholm, 12 -15 August, pp. 691- 694, 2013.

[12] J. Aikio, and D. Howe, "Extremely short external cavity laser: profilometry via wavelength tuning ", Conference of laser and electro optics, pp 484-485, 2001.
[13] Aikio, J.K., Kataja, K.J., Alajoki, T., Korioja, P., Howe, D.G., "Extremely short external cavity lasers: The use of wavelength tuning effects in near field sensing", Proceedings of SPIE - The international Society for Optical Engineering, pp. 235-245, 2002.

[14] Hoffmann, M., Voges, E., "Bulk silicon micromachining for MEMS in optical communication systems", Journal of Micromechanics and Micro Engineering, Vol. 12 pp.349-360, 2002.

[15] A. M. Abu-El-Magd, " Double Tuning of A Dual External Cavity Semi Conductor Laser For Broad Wavelength Tuning With High Side Mode Suppression", Master Thesis, McMaster University, Hamilton, 2011

[16] H. Cai, J. X. Lin, J. H. Wu, B. Dong, Y. D. Gu, Z. C. Yang, Y. F. Jin, Y. L. Hao, D. L. Kwongand A. Q. Liu, "NEMS optical cross connect (OXC) driven by optical force", 28th IEEE International Conference on Micro Electro Mechanical Systems (IEEE MEMS), 2015

[17] B. Dong, H. Cai, Y. D. Gu, Z. C. Yang, Y. F. Jin, Y. L. Hao, D. L. Kwong and A. Q. Liu, "NEMS variable optical attenuation (VOA) driven by optical force", 28th IEEE International Conference on Micro Electro Mechanical Systems (IEEE MEMS), 2015.

[18] W. M. Zhu, X. M. Zhang, T. Zhong, A. Q. Liu and M. Yu, "Micromachined optical well structure for thermo-optic switching," Applied Physics Letters, Vol. 91, No.26,261106, 2007.

[19] A. Q. Liu, A. B. Yu, M. F. Karim and M. Tang, "RF MEMS switches and integrated switching circuit," Journal of Semiconductor Technology and Science, Special issue on NANO/Microsystems Technology, Vol. 7, No.3, September, 2007.

[20] Y.M. Sabry, D. Khalil, B. Saadany, and T. Bourouina, "Silicon Micromirrors with Three-Dimensional Curvature Enabling Lensless Efficient Coupling of Free-Space Light", Light: Science \& Applications, 2, e94,2013.

[21] X. Zhu, and D. T. Cassidy, "Liquid Detection with InGaAsP Semiconductor Lasers Having Multiple Short External Cavities", Applied Optics, vol 35, pp 4689-4693, 1996.

[22] A., Fawzy, O., M., EL-ghandour, H., F., A., Hamed.,"Performance Analysis on a Dual External Cavity Tunable Laser ECTL source", Journal of Electromagnetic Analysis and Applications,Vol.7, PP. 134-139.,2015.

[23] B. Dong, J. G. Huang, H. Cai, P. Kropelnicki, A. B. Randles, Y. D. Gu and A. Q. Liu, "An all optical shock sensor based on buckled doublyclamped silicon beam", 27th IEEE International Conference on Micro Electro Mechanical Systems (MEMS 2014), San Francisco.

[24] X. Zhao, J. M. Tsai, H. Cai, X. M. Ji, J. Zhou, M. H. Bao, Y. P. Huang, D. L. Kwong and A. Q. Liu, "A nano-opto-mechanical pressure sensor via ring resonator," Optics Express, Vol 20, pp.8535-8542, 2012.

[25] J. F. Tao, H. Cai, J. Wu, J. M. Tsai, Q. X. Zhang, J. T. Lin and A. Q. Liu, "Optical wavelength signal detector via tunable micro-ring resonator for sensor applications", 26th IEEE International Conference on Micro Electro Mechanical Systems (MEMS 2013), Taipei.

[26] J.F. Tao, A. B. Yu, H. Cai, W. M. Zhu, Q.X. Zhang, J. Wu, K. Xu, J. T. Lin, ,A. Q. Liu, "Ultra-high coupling efficiency of MEMS tunable laser via 3-dimensional micro-optical coupling system" IEEE 24th International Conference on MEMS, pp. 13-16, 2011. 